\title{
Erratum: Tunneling density of states, correlation energy, and spin polarization in the fractional quantum Hall regime [Phys. Rev. B 100, 085107 (2019)]
}

\author{
Gaurav Chaudhary ${ }^{\circledR}$, Dmitry K. Efimkin, and Allan H. MacDonald \\ (Received 1 November 2019; published 14 November 2019)
}

DOI: 10.1103/PhysRevB.100.209903

The measurement idea proposed in Figs. 3(a) and 3(b) and Eq. (29) of the paper was previously conceived [1,2] and has recently [3] been thoroughly explored experimentally.

[1] H. M. Yoo, J. Jang, L. Pfeiffer, K. West, and R. C. Ashoori, Proceedings of the 22nd International Conference on Electronic Properties of Two Dimensional Systems, University Park, 2017 (Pen State University, Philadelphia, 2017), p. 21, https://sites.psu.edu/ep2ds2017/files/ 2016/06/Program_and_Abstracts_final-21w5yca.pdf.

[2] H. M. Yoo, J. Jang, L. Pfeiffer, K. West, and R. C. Ashoori, March Meeting 2018, Los Angeles, March 2018, http://meetings.aps.org/link/ BAPS.2018.MAR.F07.10.

[3] H. M. Yoo, K. W. Baldwin, K. West, L. Pfeiffer, and R. C. Ashoori, Complete spin phase diagram of the fractional quantum Hall liquid, arXiv:1910.01109. 